

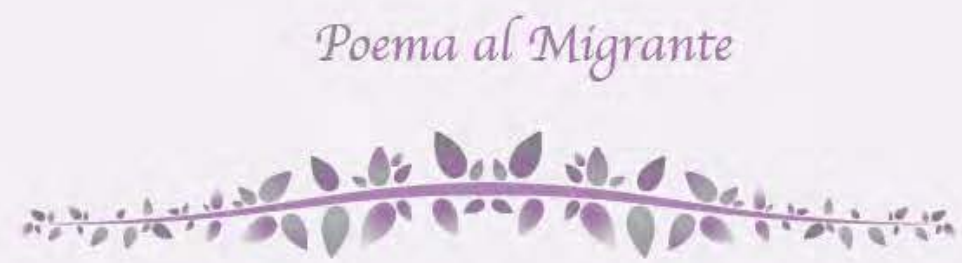

Migrante labrador de sueños

¿Qué termína? ¿Qué comienza?

Vamos cerrando camínos, abriendo nuevos rumbos, Son enigmas palpitantes, esplendor divisado.

¿Sortija del tiempo?, zcirculares eventos?

Avanzamos a otro rumbo por pedregosa senda.

Son latidos del tiempo nuevo, estupor del alma y pródigo afán.

Incendiaria disputa interna, estupefacta ventura,

Estocada de amor, Grisa, estirpe y espiga,

Soledad y orgutto, compañia y afecto,

Fugaces ventarrones del alma, extravios sonoros, Herida y lanza, sonrisa y cómplice mirada,

ráfagas de tímpo ido Vaivén del alma, brocado de recuerdos gratos, espiga de luz te llevamos dentro.

Hijo de mi esperanza, hija de la ternura, divina presencía.

T'u mirada franca, tu figura frágil es motor que empuja.

Desafía sucesos y contrariedades, invita a seguir, en marcha, siempre adelante.

Buscando del abanico de posibilidades, el que te haga más feliz. Deseando que los sueños no sean ilusión, ni flor de temporada Sino permanente canto a la vida, realización e filo de ensueño.

Todo esto pronto será recuerdo, trueno, temblor, penumbra superada. Quedó atrás el abrazo tierno, la fugaz despedida y lágrimas no enjugadas. Se rompe el dolor y el remoto silencio, en medio del frenesi de llamadas, Pronunciamos palabras, cierran ventanas, abren las puertas.

El relámpago cegador de banalidades dice presente.

Queriendo enmudecer los más hermosos sueños. 


\title{
Migración del Triángulo Norte de Centroamérica a los Estados Unidos de América
}

\author{
MANUEL ANTONIO FLORES FONSECA ${ }^{1}$ \\ ORCID: 0000-0003-4929-7354 \\ Recibido 20.05.16/Aprobado 05.08.16
}

\begin{abstract}
RESUMEN. Los países del Triángulo Norte de Centroamérica: Guatemala, El Salvador y Honduras, abarcan una extensión territorial de 242.4 mil km2 y en las últimas seis décadas han incrementado ostensiblemente su población, pasando de 6.8 millones en 1950 a 30.9 millones en el 2015, incrementándose en 4.5 veces. En la actualidad los países del Triángulo Norte generan los mayores flujos migratorios hacia los Estados Unidos de América, en el siguiente orden: El Salvador, Guatemala y Honduras, contribuyendo con alrededor del 85\% de la población de origen hispano centroamericano (siendo 4 millones de personas en el 2012) residen en ese destino. Este trabajo quiere mostrar un panorama actual de la migración internacional de los países del Triángulo Norte de Centroamérica hacia los Estados Unidos de América, siendo una sistematización de información del fenómeno migratorio de los países de la subregión, tomando en cuenta las fuentes de datos e información desde el origen, el tránsito y el destino, desde la perspectiva de la demografía. Las fuentes de información secundarias utilizadas son las bibliográficas, los informes, memorias, artículos, reportajes y otras experiencias de investigación, aunque la perspectiva demográfica conduce que las principales fuentes de datos son estadísticas y nuestro enfoque está en la búsqueda, clasificación, obtención, generación y análisis de los datos, localizadas en los países de origen, en tránsito y destino, tales como los Censos de Población, Encuestas de Hogares, Estadísticas Migratorias, Estadísticas de Aprehensión y Deportación, Encuestas Especiales de Migración y las Estimaciones de Población Migrante en tránsito y en el destino como también los no autorizados y los posibles aplicantes a programas de regularización.
\end{abstract}

Palabras Clave: Migración internacional, Emigración internacional, Centroamérica, Triángulo Norte de Centroamérica.

ABSTRACT. The countries of the Northern Triangle of Central America: Guatemala, El Salvador and Honduras, covering an area of 242,400 km2, and in the past six decades have dramatically increased their population, from 6.8 million in 1950 reached 30.9 million in 2015, increasing by 4.5 times. Today the countries of the Northern Triangle generate the largest migratory flows to the United States of America, in the following order: El Salvador, Guatemala and Honduras, contributing around 85\% of the Hispanic population Central (being 4 million people in 2012) reside in that destination. This paper wants to show a current view of international migration from the countries of Northern Central Triangle to the United States of America, being a systematization of information of migration from the countries of the subregion, taking into account the sources of data and information from the origin, transit and destination, from the perspective of demography. Secondary sources of information used are the literature,, reports, memoirs, articles, reports and other research experiences, although the demographic perspective leads to the main data sources are statistical and our focus is on searching, sorting, acquisition, generation and analysis of data, located in the countries of origin, transit and destination, such as population censuses, household surveys, migration statistics, statistics Apprehension and Removal, Special Surveys Migration and Population Estimates Migrante in transit and destination as well as unauthorized and potential applicants for regularization programs.

Keywords: International Migration, International Emigration, Central America, Triangle North Central America.

\section{Introducción}

Los países de Guatemala, El Salvador y Honduras conforman el llamado "Triángulo Norte de Centroamérica", albergan en 242.4 mil km² a 30.9 millones de personas, aunque todos de ellos se encuentran en la transición demográfica con las reducciones de la mortalidad y la fecundidad, los efectos de esa transición todavía se manifiestan en la presión demográfica, con el consecuentemente aumento de la población total y de las potencialmente activas hasta el mediano y largo plazo del presente siglo, cambios en estructura por edades que pueden ser aprovechados en el umbral del bono demográfico. A su vez estos países han tenido un lento recorrido en procura del desarrollo que se origina desde el descubrimiento, conquista y la colonización que dura más de trescientos años hasta convertirse en pequeñas naciones frágiles de dominios foráneos.

La Reforma Liberal de fines del siglo XIX, las economías de enclave, guerras intestinas, el nacimiento de estados modernos en la primera mitad del siglo XX, la introducción del capitalismo, largos periodos de dictaduras y golpes de estado, la guerra del futbol (Honduras-El

1. Máster en Población y Desarrollo, Docente Investigador del Instituto de Investigaciones Económicas y Sociales (IIES) de la Facultad de Ciencias Económicas, Administrativas y Contables (FCEAC) de la Universidad Nacional Autónoma de Honduras (UNAH), Email: mflores@,iies-unah.org, manuel.flores@unah.edu.hn 
Salvador), guerras internas por dominios de potencias extranjeras. La globalización ha significado en las últimas décadas la introducción de las políticas neoliberales, liberalización de mercados, tratados de libre comercio, unión aduanera, dolarización, privatización de entes públicos, asociaciones público-privadas, concesiones de los recursos naturales, zonas especiales de desarrollo, etc.

Por otro lado, la historia de los países del Triángulo Norte de Centroamérica ha significado a través del tiempo segmentos crecientes de poblaciones sumidas en la pobreza y la desigualdad, niveles precarios de desarrollo humano, bajos niveles de educación y acceso a la salud, problemas de gobernabilidad, falta de transparencia y corrupción, vorágine de violencia, muerte, impunidad, deterioro de los derechos humanos, invasión en territorios y deterioro del medio ambiente por industrias extractivas, etc.

En este contexto los movimientos migratorios al exterior de los países del Triángulo Norte de Centroamérica repuntaron con los conflictos armados en la región hacia la emigración en Guatemala y El Salvador desde los ochenta, pero aún con los acuerdos de paz continuaron la pobreza, la desigualdad y exclusión que se profundizaron con las políticas económicas neoliberales, que después suma a Honduras con una migración tardía, un desastre natural que los afecta a finales de los noventa y se agrega más recientemente la escalada de violencia que descansa en grupos irregulares, narcotráfico, corrupción e impunidad. No es de extrañar incluso que se produzcan nuevas manifestaciones del fenómeno como es el caso de los niños migrantes no acompañados.

Desde el inicio del milenio la política de seguridad ha invadido el tema migratorio, tanto en políticas, planes, tecnologías, restricciones, medidas que ya traspasan los países de destino hacia los países de origen y tránsito y su aplicabilidad en la subregión del triángulo norte no es la excepción, sin embargo, los flujos migratorios continúan hacia al norte y los países no pueden retener población porque no atacan las causas estructurales que influyen en la migración.

Ahora hay que esperar los resultados del Plan Alianza de la Prosperidad dirigido al Triángulo Norte que pretende entre varios objetivos atenuar la emigración. Este trabajo quiere mostrar un panorama actual de la emigración de la subregión, que aunque no es un nada nuevo evoluciona en el tiempo presentando nuevas características.

\section{Marco de referencia}

En los tiempos modernos el fenómeno migratorio se ha beneficiado por la utilización de medios de transporte y de las nuevas tecnologías de comunicación generadas en el contexto de la globalización, que favorece algunos desplazamientos de personas, pero restringe otros de acuerdo a las tendencias de los mercados mundiales. En general, los desplazamientos han tenido su origen en las desigualdades económicas y sociales, las tensiones políticas, los conflictos armados y por los desastres naturales. Cierto es que actualmente las migraciones ahora son más conocidas que en el pasado por el desarrollo de sistemas de captación, generación de conocimiento científico y desde luego su mayor visibilización en los medios de comunicación.

En el mundo hay aproximadamente mil millones de migrantes, de los cuales más de 232 millones son migrantes internacionales ( $3 \%$ de la población mundial), aunque hay que mencionar que los mayores volúmenes de migrantes se producen internamente en los países (OIM, 2015). La mayor parte de la migración se dirige hacia los países del norte, pero hay un volumen prácticamente equivalente de migración a los países del sur. Los fenómenos migratorios en el orbe actualmente tienen de contexto acontecimientos asociados a la crisis económica mundial, los generados por los desastres naturales y el cambio climático y los conflictos políticos que muchas veces desembocan en conflictos armados.

Los estados de la región se modernizan a partir de los años cincuenta con el desarrollo de la infraestructura física, creación y fortalecimiento de instituciones de los estados, diversificación de la economía, de las exportaciones y de los cultivos. Los años sesenta en la región están enmarcados con el nacimiento del Mercado Común Centroamericano (MCC) que introduce la sustitución de importaciones como modelo alternativo de desarrollo económico, mismo que tiene fisuras a raíz de la llamada "guerra del futbol" entre Honduras y El Salvador, aunque sus verdaderas causas fueron políticas en ambos países, alentadas por comportamientos xenofóbicos principalmente de la migración salvadoreña a Honduras, que inició tempranamente con los empleos agrícolas de las compañías bananeras en el norte y después se consolidó en todo el país con campesinos y otra mano de obra en la búsqueda de tierras agrícolas y otros trabajos en las ciudades en los años cincuenta y sesenta ${ }^{2}$. En los años setenta los

2. Hay diversas estimaciones sobre la emigración salvadoreña a Honduras; 300 mil (12\%) por Francisco R. R. de Sola (1969). Flores (2012) destaca que la información de los censos de población hondureños; de 1961 arroja 38 mil salvadoreños ( $75 \%$ de los extranjeros) y en 1974 se oculta el dato directo de empadronados salvadoreños, sin embargo, genera un total de 100,743 extranjeros que residían en el país, cinco años del censo, o sea en 1969 que muchos posiblemente eran salvadoreños. 
conflictos armados en Nicaragua y la inestabilidad política de gobiernos autoritarios en El Salvador, Honduras y Nicaragua generan crisis y desplazamientos internos, extra nacionales y extra regionales.

La década de los ochenta en la región Centroamericana el modelo de sustitución de importaciones llega a su agotamiento, la crisis económica y de la deuda se vuelve agobiante, repercute en el deterioro económico y social de las poblaciones, al mismo tiempo el recrudecimiento de la guerra fría se traslada a la región y genera el avivamiento de conflictos armados en Guatemala, Nicaragua y El Salvador que provoca un mayor flujo de traslados intrarregionales de refugiados y migrantes que huyen de los conflictos, a su vez se profundiza la emigración internacional hacia los Estados Unidos de América (Flores Fonseca, 2015).

En los años noventa con la implementación de los procesos de ajuste estructural de las economías que profundiza la pobreza y las desigualdades de la población los flujos emigratorios que se habían originado de los conflictos armados en la región ahora son efecto de las crisis económicas y de sus paliativos, aun cuando se culminan los procesos de paz, por lo que continúa la emigración hacia el norte que al final de la década son profundizados por los efectos económicos que generan los desastres naturales, principalmente el Huracán Mitch que afectó toda la región e hizo que los flujos de personas al exterior se visibilizaran, incluso los Estados Unidos de América aprobó un Estatus de Protección Temporal (TPS), para quienes habían ingresado a ese país antes de la ocurrencia del fenómeno climático, pero cuyo propósito directo era frenar la emigración de centroamericanos ocasionada por la destrucción, desempleo y desesperanza generada por el desastre natural.

El nuevo milenio significó en los primeros años una época de reconstrucción de la infraestructura física y económica de los países afectados por los desastres naturales, pero también la paulatina pérdida de la paz que ha ocurrido en el Triángulo Norte de Centroamérica esta vez por el recrudecimiento de la violencia ${ }^{3}$ generada por la proliferación de grupos armados irregulares (maras, pandillas, delincuentes, traficantes), la utilización de territorios por el narcotráfico y crimen organizado y los altos niveles de impunidad en la impartición de justicia.
A su vez en toda la década y media desde el inicio del siglo la región se ha visto imbuida en los procesos acelerados de globalización, políticas de liberalización de las economías, privatización de entes públicos, el establecimientos de nuevos tratados de libre comercio, unión aduanera, asociaciones público-privadas, zonas especiales de desarrollo. A su vez existen altos niveles de corrupción y poca transparencia gubernamental, que ha llevado a un deterioro del Estado de Bienestar en detrimento de grandes estratos de la población que sumidos en la pobreza y la desigualdad tienen que generar ingresos para sobrevivir en países donde la generación de empleo no alcanza los niveles que la presión demográfica exige. Aunque en el periodo los países han llevado a cabo procesos electorales, cierto es que existe desilusión en los estratos medios y bajos por su efectividad y transparencia que incluso desembocan en algunos casos en crisis de gobernabilidad.

Los países del Triángulo Norte de Centroamérica en la actualidad generan los mayores flujos de migración hacia los Estados Unidos de América, en el siguiente orden El Salvador, Guatemala y Honduras, contribuyendo con alrededor del $85 \%$ de la población de origen hispano centroamericano ( 4 de 4.7 millones de personas en el 2012) residen en ese destino (Flores Fonseca, 2015).

En Centroamérica continua los volúmenes de poblaciones que se desplazan internacionalmente (regional ${ }^{4}$ o extra-regionalmente), aunque es preciso apuntar que Costa Rica y Panamá son considerados destinos migratorios atractivos, mientras que los países del Triángulo Norte son considerados territorios productores de emigrantes, cuyo principal destino es Estados Unidos de América, poblaciones que cruzan por diferentes medios los países de tránsito, principalmente el largo territorio mexicano ${ }^{5}$ con el objetivo de salir o huir de sus países en la búsqueda del sueño americano. Las causas de la emigración de la subregión continúan siendo la búsqueda de oportunidades, principalmente económicas a través de un trabajo que permita generar ingresos, el mejoramiento de las condiciones de bienestar de las familias en su país de origen y en los últimos años el encontrar un lugar seguro, por la escalada de violencia que azota los países del triángulo norte generada por las maras, pandillas, el narcotráfico, delincuencia y la extorsión en naciones donde

3. Los países del Triángulo Norte de Centroamérica tienen de las tasas de homicidios más altas del mundo.

4. Costa Rica es atractivo principalmente para los Nicaragüenses que constituyen la mayoría de los migrantes en ese país, Panamá tiene flujos para diversificados por su economía de servicios, comercio y los generados por el Canal de Panamá. A su vez hay intercambios regionales importantes entre Honduras-El Salvador, Nicaragua-Honduras, Nicaragua-El Salvador, principalmente por motivos laborales, agrícolas y comerciales. Guatemala tiene acceso a desplazamientos temporales en el sur de México.

5. México se ha convertido en territorio difícil para los migrantes centroamericanos, principalmente del triángulo norte ya que los cruzarlo significan sortear miles de kilómetros en medios de transporte no seguros (la Bestia), eludir las detenciones de policías y de agentes migratorios que cada vez son más efectivas por la implementación de planes de seguridad, pero principalmente el encuentro con grupos irregulares que asaltan, despojan, secuestran e incluso provocan muertes. A partir del 2014 México desarrolla el "Plan Frontera Sur" que se centra en la administración y control de flujos migratorios en la frontera sur, desde una óptica de seguridad nacional. A su vez en el trayecto existe la mano amiga de grupos de protección a migrantes como el grupo Beta, las Patronas que alimentan a migrantes que transitan en trenes de carga, las iglesias que brindan refugio, información, asistencia y protección. 
aflora la impunidad. Incluso en la región ha aumentado el número de solicitantes de asilo en terceros países por causas políticas y principalmente por causas relativas a encontrar refugio de la violencia.

Los últimos acontecimientos que contextualizan a la migración de los países del triángulo norte son el endurecimiento de las políticas de seguridad de los EUA (que viene desde 2001) y que en los últimos años involucra completamente a México ${ }^{6}$ y a los países de la subregión con el combate al narcotráfico y seguridad, pero que invade el ámbito migratorio con sus efectos de la implementación de grandes recursos en la militarización de territorios, tecnologías, estaciones y agentes migratorios y que llega a incluso querer impedir la salida de personas de sus países de origen.

En los últimos años y principalmente en el 2014 se produjo una oleada de niños migrantes no acompañados que fueron detenidos por las autoridades estadounidenses en la frontera, principalmente en Texas, convirtiendo el fenómeno "crisis humanitaria" en un evento con un alto contenido mediático que fue utilizado por los gobiernos y organismos para solicitar recursos para su atención. El gobierno estadounidense recientemente está ejecutando nuevas ventanas de aplicación migratoria como el Plan DAPA, DACA y DACA-Plus que comprende regularizar a poblaciones que llegaron a ese país en la niñez antes de determinada fecha, ser joven, contar con ciertos niveles de educación, tiempo de residencia, no tener delitos graves ni de ser amenaza para seguridad.

El nuevo esquema de implementación de las políticas del gobierno estadounidense en la región es el llamado "Plan de Alianza para la Prosperidad, PAP" que implica la asignación del Congreso de 750 millones dólares de ayuda a los países del Triángulo Norte de Centroamérica, que incluye desde luego recursos para el desarrollo, programas de gobierno, narcotráfico, seguridad, en tratar de atacar las causas de la pobreza, violencia e implícitamente la emigración hacia el norte.

\section{Materiales y métodos}

Este trabajo quiere mostrar algunos elementos de la migración internacional de los países del Triángulo Norte de Centroamérica, que incluye los territorios de Guatemala, El Salvador y Honduras hacia los Estados Unidos de América. El objetivo de esta investigación es sistematizar información de la migración de los países del triángulo norte de Centroamérica hacia los Estados Unidos de América, tomando en cuenta las fuentes de datos e información desde los países de origen, de tránsito y destino, desde la perspectiva de la demografía.

Las fuentes de información utilizadas en este trabajo son las bibliográficas, los informes, memorias, artículos, reportajes y otras experiencias de investigación, aunque la perspectiva demográfica principal es enfocada a las fuentes estadísticas en primer lugar, a las metodológicas de las fuentes de datos y finalmente en el análisis de datos.

Las principales fuentes de datos que se utilizan en esta investigación son del país de origen, tránsito y destino, entre ellas están los Censos de Población (CP Guatemala, El Salvador, Honduras, México y EUA), las Encuestas de la Comunidad Americana (ACS), estadísticas de la Oficina de Aduanas y Protección Fronteriza de los EUA (US Customs and Border Protection), Oficina de Inmigración del Departamento de Seguridad Nacional (DHS) de EUA, la Encuesta sobre Migración en la Frontera Sur de México (EMIF Sur), datos de la Secretaría de Gobernación de México (SEGOB), Direcciones o Institutos Nacionales de Migración de los países del Triángulo Norte de Centroamérica (DGM, DGME, INM), las Encuestas especiales con módulos de Migración, Estudios de Migración de Tanques de Pensamiento (MPI, PEW Research), otros.

\section{Discusión de resultados}

\subsection{Tendencias demográficas de los países del Triángulo Norte de Centroamérica}

En el Triángulo Norte de Centroamérica vive una población alrededor de treinta y uno millones de personas y los países que más contribuyen demográficamente son Guatemala y Honduras, mientras El Salvador lo hace en menor medida.

Todos los países están inmersos en la transición demográfica, esto como consecuencia del descenso de la mortalidad antes que la fecundidad, desde antes de los años cincuenta, mientras que la última su descenso fue generalmente tardío y lento, aunque hay que tener en cuenta las especificidades de cada uno de los tres países de la región del Triángulo Norte.

Actualmente con las transformaciones poblacionales que experimentan los países de Triángulo Norte centroamericano, originadas de la transición demográfica, el crecimiento de la población continuará hasta el largo plazo, a su vez niveles de población potencialmente activa como nunca en su historia, que significa grandes contingentes de

6. El Plan Frontera Sur que implementa México ha aumentado extraordinariamente las detenciones en ese país para que no lleguen hasta la frontera norteamericana. 
fuerza de trabajo que aumentará hasta el mediano plazo, pero el hecho más fundamental es la ocurrencia del bono demográfico en el futuro cercano -El Salvador alcanzará el umbral del bono entre 2012 al 2040, Honduras entre los años 2018 al 2043 y Guatemala entre los años 2029 al 2054- (Flores Fonseca, 2015). El bono demográfico es una oportunidad que desde el comportamiento de la población puede contribuir con el crecimiento económico y puede ser aprovechado construyendo políticas adecuadas en la economía, educación y la gobernabilidad. Otro escenario es que la oportunidad transcurra en el tiempo sin acciones de política eficientes de aprovechamiento, aunque siempre se obtienen beneficios económicos, se pueden producir desajustes por las crecientes demandas económicas, empleo, educativas, sociales, de infraestructura, etc., que se originan del crecimiento inercial de la población, en tanto la migración al exterior se convierte en una válvula de escape cuando los desajustes de las sociedades nacionales afectan principalmente a los jóvenes y adultos jóvenes en la búsqueda de oportunidades.

\subsection{Migración desde el país de orígen}

\subsubsection{Censos de población de los países del Triángulo Norte de Centroamérica}

Los censos de población y vivienda (CPV) son fotografías en un momento dado de las condiciones demográficas, sociales y económicas de personas referidas a un territorio determinado, tienen el principio de universalidad que permite abarcar a toda la población, pero incluso también se puede ahondar respecto a las características de los hogares que tienen personas en el exterior del país, o rescatar algunas características de los migrantes, generalmente de los inmigrantes siempre se ha recogido información (nacidos en el extranjero es la aproximación), pero de los emigrantes la situación no ha sido tan fluida, ya que no se encuentran residiendo en el lugar de empadronamiento y la información solo puede ser suministrada por sus familiares.

Los censos de los países centroamericanos que conforman el Triángulo Norte no se ha realizado en el mismo año, como proponen las recomendaciones censales de Naciones Unidas, para el caso en Guatemala el último se realizó en el 2002, en El Salvador en el 2007 y en Honduras en 2013.

El Censo de Población y Vivienda del 2007 de El Salvador estimó un total de 155,919 hogares con emigrantes en el exterior que representa un $11.29 \%$ del total de hogares, a su vez se recogió información de 261,587 emigrantes, de los cuales 155,919 son hombres $(59.6 \%)$ y 93,925 mujeres (36.0\%) e ignorados 11,743 (4.4\%), que salieron de área urbanas un $57.4 \%$ y áreas rurales el $42.6 \%$. En el censo de población también se recogió información de los nacidos en el exterior, con la pregunta de "Dónde vivía su madre cuando nació", que suman un total de 37,820 personas, representando el $0.65 \%$ del total, a su vez se formuló la pregunta "Desde cuando vive en esta cabecera o cantón" (año) y “donde vivía antes", en otro país (DIGESTYC D. G., 2009).

En el caso de Honduras se realizó el Censo de Población y Vivienda en el 2013, los resultados indican que 128,619 personas viven en otro país en los últimos diez años, de los cuales el $66.1 \%$ son hombres y $33.9 \%$ son mujeres, en tanto 90,675 personas residen en los Estados Unidos de América, 9,008 en España, en México 3,765, en Guatemala 1,008 y en el Salvador 796 y el resto que son 4,475 en otros países del mundo, hay que indicar que 18,892 no indicaron el país de residencia. Los nacidos en otro país pero que residen en Honduras suman un total de 36,853 personas, que representa solamente un $0.44 \%$ del total de la población.

Guatemala en su último Censo de Población y Vivienda que se llevó a cabo en el 2002, determinó que en 132,228 hogares al menos una persona se fue a vivir permanentemente a otro país en los últimos diez años $(49 \%$ del área urbana y $51 \%$ del área rural), representando el 6\% del total de hogares. En la inmigración el censo recogió información de los nacidos en el extranjero, mismos que suman 49,966 personas que la mitad residía en la capital de la república, a su vez se empadronó a 27,164 personas (nacionales y extranjeros) que residían en el extranjero seis años antes del censo, en la fecha de los acuerdos de paz (INE, 2003).

\subsubsection{Encuestas especiales de los países del Triángulo} Norte de Centroamérica.

Las Encuestas Nacionales de Condiciones de Vida (ENCOVI) son poderosos instrumentos estadísticos que han sido diseñados para que los países puedan recoger información sociodemográfica sobre las condiciones de la vivienda, acceso a servicios, demografía, educación, salud, empleo, ingresos y gastos, explotación agropecuaria, entre otros aspectos. La ENCOVI-2014 de Guatemala estima que en el $2.6 \%$ de los hogares, algún(os) miembro(s) emigró a otro país en los últimos cinco años con fines de permanencia, incluso en mayor porcentaje del área rural. La ENCOVI-2004 de Honduras, es una fuente de gran tamaño que además de sus objetivos fundamentales auscultó sobre migración interna (absoluta y reciente) y de la internacional (inmigración y emigración), en este último tema la ENCOVI de un total de 1,437,089 hogares estima 168,319 hogares con emigrantes internacionales $(11.7 \%)$ y un total de 254,530 emigrantes internacionales $(61.8 \%$ hombres y $38.2 \%$ mujeres). El Salvador no ha realizado las encuestas de condiciones de vida. 
Las Encuestas de Hogares y Propósitos Múltiples (EHPM) también son instrumentos de medición que además de recoger información sociodemográfica y económica de los países su principal objeto ha sido la medición de la fuerza laboral y los niveles de pobreza. En los países de la región, en El Salvador la EHPM 2014 estima un total de 379,353 personas en el extranjero, un total de 350,481 hogares reciben remesas que representa un $20.4 \%$ del total de hogares, en el área urbana $17.92 \%$ y en el área urbana $24.90 \%$, las población que reciben remesas suman 1,294,912 personas (DIGESTYC, 2015). En Honduras en las EPHPM del 2006 y 2010 se diseñaron módulos especiales para medir la migración interna e internacional, en la encuesta del 2006 se estimó un total de 179,051 hogares con emigrantes internacionales que representan el $11.3 \%$ del total de hogares, a su vez se estima 246,620 emigrantes internacionales $(70.4 \%$ hombres y $29.6 \%$ mujeres). En la encuesta del 2010 el modulo estima un total de 168,786 hogares con emigrantes internacionales $(9.7 \%$ del total) y un total de 238,669 emigrantes internacionales. En Guatemala existen las Encuestas de Empleo e Ingresos que incluye los elementos de una encuesta de hogares, no se hacen preguntas de migración, si de recibo de remesas.

\subsubsection{Estadísticas de migración de los países del} Triángulo Norte de Centroamérica

Las direcciones o institutos de migración de los países del Triángulo Norte de Centroamérica recogen información de las entradas y salidas de las personas en sus fronteras terrestres, áreas y marítimas. Con datos de sus informes se construyó el Cuadro No. 1 que presenta los totales de los movimientos migratorios, aunque nos da una idea de los flujos, es de mayor utilidad cuando se estima las entradas y las salidas para los nacionales de los países que permite estimar los diferenciales de los flujos. El Salvador presenta un nivel de entradas y salidas superior a los demás países de la subregión.

Cuadro $\mathrm{N}^{\circ}$ I. Movimientos migratorios de los países del Triángulo Norte de Centroamérica, 2012-2014

\begin{tabular}{|c|l|r|r|c|} 
País & Movimiento & 2012 & 2013 & 2014 \\
\hline \multirow{2}{*}{ Guatemala } & Entradas & $1,892,836$ & $2,222,258$ & $2,582,545$ \\
\cline { 2 - 5 } & Salidas & $1,951,836$ & $2,095,223$ & $2,372,207$ \\
\hline \multirow{2}{*}{ El Salvador } & Entradas & $3,820,248$ & $3,879.689$ & $3,672,753$ \\
\cline { 2 - 5 } & Salidas & $3,885,360$ & $3,947,849$ & $3,817,202$ \\
\hline \multirow{2}{*}{ Honduras } & Entradas* & $1,406,327$ & $1,515,713$ & \\
\cline { 2 - 5 } & Salidas** & 593,551 & 610,698 & \\
\hline
\end{tabular}

Fuente: Fuente: Institutos de Migración de Guatemala y El Salvador, Anuario Estadistico INE Honduras. *Solo incluye a extranjeros **Solo incluye a los hondureflos

\subsection{Migración desde el país en tránsito}

\subsubsection{Censos de población de México}

México es el país de tránsito más significativo para la migración de los países del Triángulo Norte de Centroamérica hacía los Estados Unidos de América, se cruza de sur a norte en recorridos que implican miles de kilómetros en territorios con climas extremos utilizando diferentes medios de movilización donde el migrante es altamente vulnerable de ser víctima de vejámenes de grupos delictivos o la detención de parte de las autoridades nativas cuando la migración se hace de manera irregular.

El censo de población y vivienda de México del 2010 se encontró solamente 33,216 personas que nacieron en los países del Triángulo Norte de Centroamérica, que fueron empadronadas y que su estadía en el país de tránsito que ya es considerada de destino (Cuadro $\mathrm{N}^{\circ} 2$ ). Guatemala tiene la mayor población extranjera en México de los países de la subregión de estudio e inclusive tiene mejores facilidades migratorias de estadía temporal como país vecino. Aunque es preciso apuntar la existencia de una población flotante cuyo objetivo migratorio es desde luego los Estados Unidos, pero que algunas veces alargan su estadía en el país de tránsito desempeñando alguna actividad generadora de ingresos de manera informal para sobrevivir o continuar el viaje.

Cuadro $\mathrm{N}^{0}$ 2. México: Población nacida en los países del Triángulo Norte de Centroamérica según Censos de Población y Vivienda, 2000 y 2010

\begin{tabular}{|c|c|c|}
\hline País & $\begin{array}{c}\text { Censo de población } \\
2000\end{array}$ & $\begin{array}{c}\text { Censo de población } \\
2010\end{array}$ \\
\hline Guatemala & 23,957 & 35,322 \\
\hline El Salvador & 5,537 & 8,088 \\
\hline Honduras & 3,722 & 10,991 \\
\hline Total & 33,216 & 54,401 \\
\hline Fuente: INEGI: XII Censo General de Población $y$ Vivienda 2000: Censo de Poblacióny Vivienda 2010
\end{tabular}

\subsubsection{Encuesta sobre migración en la frontera sur de México (EMIF Sur)}

La encuesta EMIF Sur tiene por objeto el conocimiento del flujo migratorio laboral que cruza por la frontera entre Guatemala y México, con el propósito de trabajar en México o Estados Unidos, así como de la población migrante que utiliza el territorio mexicano como país de tránsito y que es devuelta a Guatemala, Honduras y El Salvador por las autoridades migratorias mexicanas o estadounidenses. 
Entre Guatemala y México predominan los flujos transfronterizos y circulares asociados al ámbito laboral, para el 2013 el monto del flujo con sentido sur-norte (procedentes de Guatemala) fue de 754 mil eventos, mayor que el flujo norte-sur (procedentes de México o Estados Unidos) que fue de 698 mil eventos, entran más personas que las que regresan a su país de origen. En general, a través del tiempo en los flujos aumenta su volumen y crece su ritmo, siendo de ámbito laboral, principalmente joven y con bajos niveles de escolaridad.

Los flujos migratorios de centroamericanos devueltos por autoridades migratorias de México y de Estados Unidos, permiten hacer estimaciones indirectas de la magnitud del flujo de tránsito por México. La EMIF Sur capta el flujo de migrantes de los países del triángulo norte de Centroamérica quienes son detenidos por las autoridades mexicanas y devueltos a sus respectivos países, mayoritariamente entran a México sin documentos y con el objetivo de llegar a Estados Unidos (Cuadro $\mathrm{N}^{\circ} 3$ ). Para el año 2013 se estimó un total de 73 mil eventos, con una participación similar entre personas guatemaltecas 29 mil eventos, hondureñas 29 mil eventos y salvadoreños 14 mil eventos. De EUA se registran 102 mil eventos de deportación de los países de Centroamérica, Guatemala 49 mil, El Salvador 21 mil y Honduras 32 mil eventos, que son más los que llegan a su destino del norte (Colegio de la Frontera Norte, Secretaría de Gobernación, Secretaría de Trabajo y Previsión Social, Secretaría de Relaciones Exteriores, 2014).

Cuadro $\mathrm{N}^{\circ}$ 3. México: monto anual de los flujos de personas de países del Triángulo Norte de Centroamérica devueltos por las autoridades migratorias de México y de Estados Unidos de América, 2009-2013, en miles

\begin{tabular}{|c|c|c|c|c|c|c|}
\hline \multirow{1}{*}{ País } & $\begin{array}{c}\text { País de } \\
\text { devolución }\end{array}$ & 2009 & 2010 & 2011 & 2012 & 2013 \\
\hline \multirow{3}{*}{ Guatemala } & México & 28 & 28 & 30 & 35 & 29 \\
\cline { 2 - 7 } & EUA & 31 & 29 & 30 & 40 & 49 \\
\hline \multirow{2}{*}{ El Salvador } & México & 10 & 10 & 8 & 12 & 14 \\
\cline { 2 - 7 } & EUA & 23 & 21 & 17 & 19 & 21 \\
\hline \multirow{2}{*}{ Honduras } & México & 23 & 23 & 17 & 27 & 29 \\
\cline { 2 - 7 } & EUA & 25 & 24 & 22 & 32 & 32 \\
\hline \multirow{2}{*}{ Fuente: Datas recopilados de la Encuesta de Migración de la Frontera Sur de México, EMIF-Sur, 2013 }
\end{tabular}

\subsubsection{Estadísticas migratorias de México}

En México como principal país de tránsito de la migración de la subregión hacia los Estados Unidos, la Secretaría de Gobernación de México (SEGOB) a través de la Subsecretaría de Población, Migración y Asuntos Religiosos se encarga entre otras atribuciones de integrar, generar y publicar la estadística oficial sobre la movilidad y migración internacional en ese país, a partir de los registros administrativos.

Guatemala y El Salvador presentan las mayores entradas áreas de los tres países que suman $124.6 \mathrm{mil}$, mientras Honduras y El Salvador han solicitado un mayor número de casos de residencias como refugiados. En los casos de presentación ante autoridades migratorias, también en menores, devoluciones, retornos asistidos de menores tanto Guatemala como Honduras llevan la delantera en estos eventos, la excepción con El Salvador son los casos de eventos de retorno asistido de mayores de edad (Cuadro $\mathrm{N}^{\circ} 4$ ).

Cuadro $\mathrm{N}^{\circ}$ 4. México: principales estadísticas migratorias de personas de los países del Triángulo Norte de Centroamérica, 2014

\begin{tabular}{|c|c|c|c|c|}
\hline Categoría & Guatemala & $\begin{array}{c}\text { El } \\
\text { Salvador }\end{array}$ & Honduras & Total \\
\hline Entradas aéreas & 67,136 & 37,347 & 20,190 & 124,673 \\
\hline $\begin{array}{l}\text { Tarjeta residencia } \\
\text { temporal (TRT) }\end{array}$ & 641 & 487 & 564 & 1,692 \\
\hline $\begin{array}{l}\text { Tarjeta residencia } \\
\text { permanente (TRP) }\end{array}$ & 2,640 & 1,210 & 2,269 & 6,119 \\
\hline $\begin{array}{l}\text { Extranjeros documentados } \\
\text { residencia permantente } \\
\text { como refugiado }\end{array}$ & 12 & 93 & 146 & 251 \\
\hline $\begin{array}{l}\text { Tarjeta Visitante por } \\
\text { razones humanitarias } \\
\text { (TVRH) }\end{array}$ & 63 & 115 & 305 & 483 \\
\hline $\begin{array}{l}\text { Eventos de } \\
\text { presentación ante } \\
\text { autoridades migratorias }\end{array}$ & 47,794 & 23,131 & 47,521 & 118,446 \\
\hline $\begin{array}{l}\text { Eventos de menores } \\
\text { presentados ante } \\
\text { autoridades migratorias }\end{array}$ & 7,973 & 4,885 & 9,661 & 22,519 \\
\hline $\begin{array}{l}\text { Extranjeros devueltos } \\
\text { por autoridad migratoria }\end{array}$ & 42,808 & 19,800 & 41,661 & 104,269 \\
\hline $\begin{array}{l}\text { Eventos de } \\
\text { retorno asistido }\end{array}$ & 15,933 & 36,201 & 33,517 & 85,651 \\
\hline $\begin{array}{l}\text { Eventos de retorno } \\
\text { asistido menores }\end{array}$ & 6,196 & 3,758 & 7,967 & 17,921 \\
\hline
\end{tabular}

Fuente: Bolettin Mensual de Estadisticas Migratorias 2014, UPM-SEGOB, Merico

\subsubsection{Estimaciones de migración en tránsito por} México

Para México el flujo de migración centroamericana en tránsito irregular por su territorio hacia Estados Unidos reviste gran importancia y ha llevado a realizar estimaciones con solidez estadística y metodológica. El estudio del Instituto Tecnológico Autónomo de México (ITAM) destaca que las estimaciones realizadas por Berumen y otros y las de Rodríguez y otros (Cuadro $N^{\circ} 5$ ) el volumen del flujo presentó una tendencia incremental 
desde mediados de los noventa hasta 2005, año en que alcanzó el máximo histórico, con un volumen entre 390,000 y 430,000 migrantes. Entre 2006 y 2009, hubo una caída pronunciada de cerca de $70 \%$, mientras que en 2010 y 2011 se observó una estabilización. Las estimaciones preliminares para 2012 evidencian un repunte de los flujos de cuando menos un $40 \%$ (ITAM, 2014). Aun así aunque hay variaciones en el tiempo los datos muestran un flujo relevante de migrantes centroamericanos en tránsito irregular por México.

\section{Cuadro $\mathrm{N}^{0}$ 5. México: estimaciones de migrantes centroamericanos en tránsito irregular hacia los Estados Unidos, 1995-2012, en miles}

\begin{tabular}{|c|c|c|}
\hline Año & Berumen 2012 & Rodriguez 2013 \\
\hline 1995 & 171 & 200 \\
\hline 1996 & 165 & 212 \\
\hline 1997 & 137 & 175 \\
\hline 1998 & 197 & 236 \\
\hline 1999 & 241 & 273 \\
\hline 2000 & 217 & 237 \\
\hline 2001 & 194 & 214 \\
\hline 2002 & 177 & 196 \\
\hline 2003 & 227 & 240 \\
\hline 2004 & 286 & 324 \\
\hline 2005 & 388 & 433 \\
\hline 2006 & 303 & 320 \\
\hline 2007 & 186 & 203 \\
\hline 2008 & 145 & 159 \\
\hline 2009 & 119 & 137 \\
\hline 2010 & 112 & 132 \\
\hline 2011 & 109 & 124 \\
\hline 2012 & & 183 \\
\hline
\end{tabular}

Fuente: Estimaciones de Rodriguez, et al 2001 y 2013; Berumen, et al. 2012. Tomado de ITAM. Migración Centroamericana en Tránsito por México hacia Estados Unidos: Diagnóstico y Recomendaciones, 2014

\subsection{Migración desde el país de destino}

\subsubsection{Censos de población de los Estados Unidos de} América

Los censos de población de los Estados Unidos de América tienen una religiosa elaboración cada diez años que hace que esta operación estadística sea la mejor fotografía de la población de ese país y de los residentes nacidos en otros países que viven en ese país del norte de América.
Los países del Triángulo Norte de Centroamérica en la actualidad generan los mayores flujos de migración hacia los Estados Unidos de América, consecuentemente en los datos censales tienen los mayores residentes en el siguiente orden El Salvador, Guatemala y Honduras, contribuyendo con alrededor del $83.2 \%$ de la población de origen hispano centroamericana (3.3 de 4 millones de personas en el 2010 ) residen en ese destino (Cuadro $N^{\circ} 6$ ). Los censos de población muestran a largo plazo el impresionante aumento entre las décadas de los ochenta y noventa y en el nuevo siglo, en el 2010 representaría el $11.8 \%$ de la población que vive en la subregión.

\section{Cuadro $\mathrm{N}^{0}$ 6. EUA: población de origen hispano de los países del Triángulo Norte de Centroamérica}

\begin{tabular}{|c|c|c|c|c|}
\multirow{2}{*}{ Años } & \multicolumn{3}{|c|}{ Países } & \multirow{2}{*}{ Total } \\
\cline { 2 - 4 } & Guatemala & El Salvador & Honduras & \\
\hline 1960 & 5,381 & 6,310 & 6,503 & 18,194 \\
\hline 1970 & 17,356 & 15,717 & 19,118 & 52,191 \\
\hline 1980 & 63,073 & 94,447 & 39,154 & 196,674 \\
\hline 1990 & 225,739 & 465,433 & 108,923 & 800,095 \\
\hline 2000 & 372,487 & 655,165 & 217,569 & $1,245,221$ \\
\hline 2010 & $1,044,209$ & $1,648,968$ & 633,401 & $3,326,578$ \\
\hline
\end{tabular}

Fuente: Elaboración con datos de la Oficina del Censo de los EUA, 2012

\subsubsection{Encuestas de la Comunidad Americana (ACS)}

La Encuesta de la Comunidad Americana (ACS) de los Estados Unidos de América, es una de Encuesta de Hogares realizada a nivel nacional anualmente, con una muestra de gran tamaño incluye todo el territorio estadounidense, la convierte en una de las principales fuentes de información de la población, condiciones de vida, sociales y económicas de los residentes en ese país (nativos y población extranjera residente). Las ACS son instrumentos estadísticos que utilizan el concepto de residencia actual (viven o se quedan por más de dos meses) en comparación de los datos censales que utilizan el de residencia habitual, y a partir de los últimos años reemplaza al cuestionario extendido de los censos de población.

En esta investigación las encuestas utilizadas son las correspondientes al periodo del 2001 al 2014, accesada a través de IPUMS (Ruggles, Genadek, Goeken, Grover, \& Sobek, 2015). En la ACS hay dos preguntas de interés para los fines de nuestra investigación, la primera es acerca del "origen hispano" de la persona, donde se incluye en el caso específico, "las personas de origen", en este caso de países del triángulo norte de Centroamérica (que no necesariamente nacieron en Guatemala, El Salvador y 
Honduras), pero declaran su pertenencia de origen de esos países. En la segunda pregunta, que es el "lugar de nacimiento", que cuando indica el país especifico siendo propiamente el mejor acercamiento de los emigrantes, ya que implica generalmente un traslado obligatorio ${ }^{7}$.

La serie de datos de población de origen y nacida en los países del Triángulo Norte de Centroamérica que recoge la ACS en más de una década es un reflejo del comportamiento migratorio de la subregión, donde los Salvadoreños encabezan la primacía en ambas categorías de poblaciones y es fiel reflejo de la historia desde los años ochenta que llevó a miles de personas abandonar su país principalmente por la guerra civil, después sigue Guatemala con una migración orientada a la búsqueda de oportunidades y la de Honduras que tardíamente ha sido más reflejo de efecto de la crisis económica, desastres naturales y en los últimos años a la violencia que azota a los tres países.

En el 2014 se estima con la ACS unas 4.2 millones de personas que mencionan como origen los tres países del triángulo norte y 2.8 millones de personas que nacieron en esos países y que residen en los Estados Unidos de América. A su vez es visible la diferenciación de la estructura por edades en las primeras edades para las poblaciones de origen y el efecto de cúspide en las edades de la juventud en las poblaciones de nacidos, con diferencias por nacionalidad originada por su historia migratoria (Gráfico $\mathrm{N}^{\circ} 1$ y 2).

Una forma de aproximarse a los flujos migratorios de los países del triángulo norte de Centroamérica en los Estados Unidos se puede realizar a través de las ACS, al estimarse de las personas nacidas en la subregión aquellas que inmigraron recientemente, el mismo año o el anterior, claramente se notan el incremento de flujos de los países, principalmente de El Salvador y Honduras (Cuadro $\mathrm{N}^{\circ} 7$ ).
Gráfico $N^{\circ} 1$. Población de origen y uacida en los países del Triángulo Norte de Centroamérica, 2001-2014

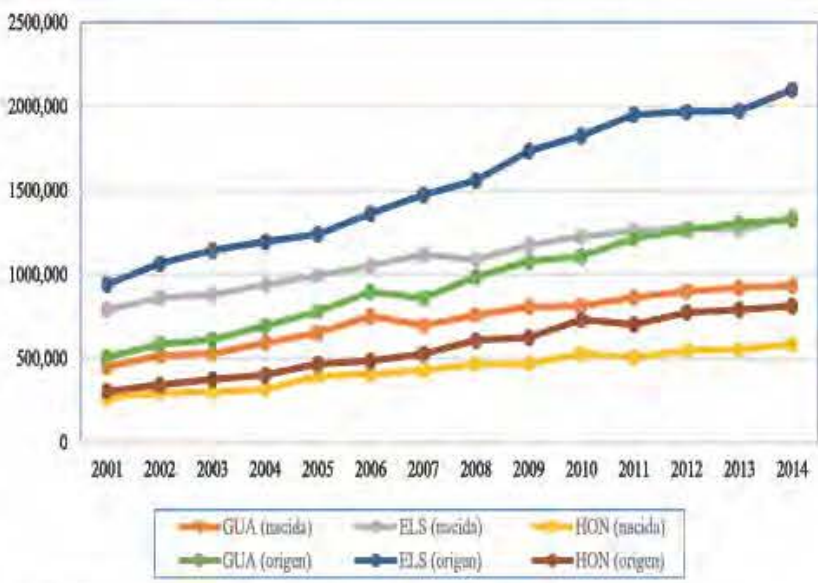

Fuente: Elahoración propia en base a datas ACS 2001-2014 en IPUMS

Gráfico $N^{\circ} 2$. EUA: población de origen y nacida en los países del Triángulo Norte de Centroamérica por grupos de edad, 2013

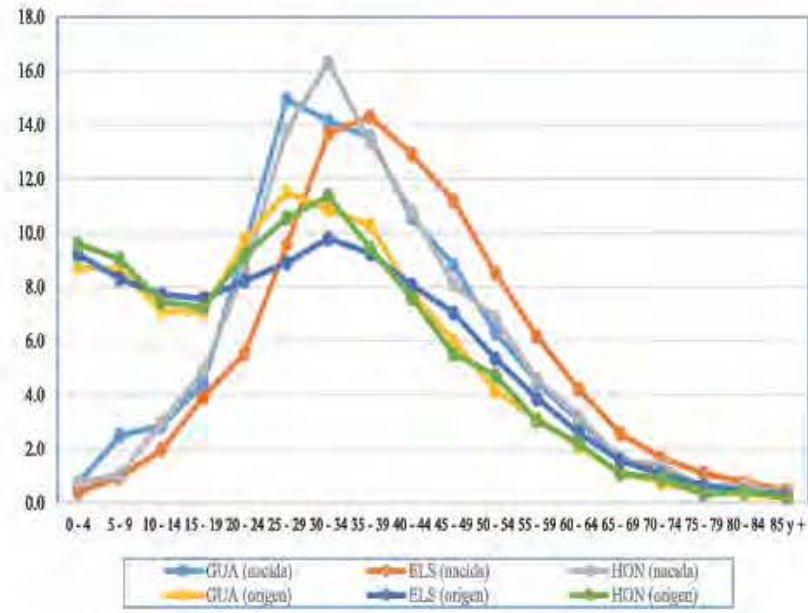

Fuente: Elaboración propia en tare a datas ACS 2013 en IPUMS

Cuadio $\mathrm{N}^{0} 7$. EUA: nacidos en páses del Triángulo Norte de Centroamérica según año de inmigración reciente, 2010-2014

\begin{tabular}{|c|c|c|c|c|c|c|}
\hline País & Año de Inmiggnción & 2010 & 2011 & 2012 & 2013 & 2014 \\
\hline \multirow{2}{*}{ Guatemala } & Mismo año & 12,722 & 7,710 & 13,617 & 15,405 & 18,787 \\
\cline { 2 - 7 } & Año anterior & 20,152 & 18,859 & 14,498 & 20,194 & 22,788 \\
\hline \multirow{2}{*}{ El Salvador } & Mismo año & 10,378 & 9,835 & 12,162 & 14,797 & 19,206 \\
\hline \multirow{2}{*}{ Honduras } & Año anterior & 19,883 & 20,803 & 17,306 & 22,607 & 37,369 \\
\cline { 2 - 7 } & Mismo año & 5,524 & 5,697 & 12,143 & 10,965 & 24,699 \\
\cline { 2 - 7 } & Año anterior & 12,658 & 12,936 & 9,663 & 15,963 & 27,576 \\
\hline \multirow{2}{*}{ Total } & Mismo año & 28,624 & 23,242 & 37,922 & 41,167 & 62,692 \\
\hline & Año anterior & 52,693 & 52,598 & 41,467 & 58,764 & 87,733 \\
\hline
\end{tabular}

Fuente: Elaboración propia en base a datos ACS 2010-2014 en IPUMS

7. Incluye los que nacen en Guatemala, El Salvador y Honduras, aunque se identifican con el origen de ellos o de sus padres. 


\subsubsection{Estadisticas de la Oficina de Inmigración del Departamento de Seguridad Nacional}

En el fenómeno migratorio otro elemento a considerar son las estadísticas que se generan en el país de destino en relación a la entrada, regularización y devolución de poblaciones que desean ingresar para residir o trabajar en él, permitiendo conocer las magnitudes de cada categoría (United States Department of Homeland, 2015). La cantidad de personas que cada año están obteniendo su estatus de residente legal en los Estados Unidos de América los nativos de la subregión no es tan elevada (Cuadro $\mathrm{N}^{\circ} 8$ ) y es fiel detalle de un proceso de regularización donde influye el tiempo de llegada, las redes y el acceso a la información.

Cuadro $\mathrm{N}^{0}$ 8. EUA: personas que están obteniendo estatus de residente permanente legal * nacidos en países del Triángulo Norte de Centroamérica, años físcales 2008-2013

\begin{tabular}{|c|c|c|c|c|}
\multirow{2}{*}{ Años } & \multicolumn{3}{|c|}{ Países } & \multirow{2}{*}{ Total } \\
\cline { 2 - 4 } & Guatemala & El Salvador & Honduras & \\
\hline 2008 & 16,182 & 19,659 & 6,540 & 42,381 \\
\hline 2009 & 12,187 & 19,909 & 6,404 & 38,500 \\
\hline 2010 & 10,467 & 18,806 & 6,448 & 35,721 \\
\hline 2011 & 11,092 & 18,667 & 6,133 & 35,892 \\
\hline 2012 & 10,341 & 16,256 & 6,884 & 33,481 \\
\hline 2013 & 10,224 & 18,260 & 8,898 & 37,382 \\
\hline
\end{tabular}

Fuente: Elaborado de cifras del 2013 Yearbook of Immigration Statistics. US. Department of Homeland Security.

*Los residentes permanentes legales ( $L P R$ ) son las personas que cuenten con residencia permanente legal en los Estados Unidos. También son conocidos como receptores "tarjeta verde"

A su vez de la regularización se encamina al proceso de asimilación de los nativos de la subregión, que llevaría a la obtención de la residencia y la naturalización para convertirse en ciudadanos de los Estados Unidos para tener mejores oportunidades para él y su familia. Hay diferencias según el país de origen que influenciada de la migración de mayor data, principalmente de los nativos de El Salvador (Cuadro $\mathrm{N}^{\circ}$ 9).

Cuadro $\mathrm{N}^{\circ}$ 9. EUA: personas naturalizadas* nacidos en países del Triángulo Norte de Centroamérica, años fiscales 2008-2013

\begin{tabular}{|c|c|c|c|c|}
\multirow{2}{*}{ Años } & \multicolumn{3}{|c|}{ Países } & \multirow{2}{*}{ Total } \\
\cline { 2 - 4 } & Guatemala & El Salvador & Honduras & S1,677 \\
\hline 2008 & 17,087 & 35,796 & 8,794 & 61,604 \\
\hline 2009 & 8,619 & 18,927 & 4,858 & 32,404 \\
\hline 2010 & 5,375 & 10,343 & 3,056 & 18,774 \\
\hline 2011 & 7,285 & 13,834 & 3,980 & 25,099 \\
\hline 2012 & 8,797 & 16,685 & 5,294 & 30,776 \\
\hline 2013 & 9,530 & 18,401 & 5,462 & 33,393 \\
\hline
\end{tabular}

Fuente: Elaborado de cifras del 2013 Yearbook of Immigration Statistics. U.S.Department of Homeland Security.

"Naturalizaciones se refieren a personas mayores de 18 años que se convierten en ciudadanos de los Estados Unidos. La mayorra de los residentes legales permanentes son elegibles para solicitar la naturalización dentro de los cinco años después de obtener el estatus de LPR
Las admisiones como no inmigrantes incluyen los eventos de entrada a los Estados Unidos por un periodo corto de tiempo, principalmente por turismo, visitas a familiares u otro motivo. El flujo de entradas oscila en un rango entre cuatrocientos y quinientos mil eventos para los tres países, diferencialmente explicada por diversas razones entre ellas el número de población de origen y nacida en los países, los residentes legales y naturalizados, llegada al país de destino, y otras características como una mayor población con posibilidades de realizar turismo, comercio o actividad laboral, entre otras. Los mayores valores de los no inmigrantes corresponden a Guatemala, Honduras y El Salvador en ese orden (Cuadro $\mathrm{N}^{\circ} 10$ ).

Cuadro $\mathrm{N}^{0} 10$. EUA: admisiones de No inmigrante* (solo I-94) de ciudadanos de países del Triángulo Norte de Centroamérica, años fiscales 2008-2013

\begin{tabular}{|c|c|c|r|c|}
\multirow{2}{*}{ Años } & \multicolumn{3}{|c|}{ Países } & \multirow{2}{*}{ Total } \\
\cline { 2 - 4 } & Guatemala & El Salvador & Honduras & \\
\hline 2008 & 224,030 & 164,984 & 139,103 & 528,117 \\
\hline 2009 & 204,944 & 141,428 & 137,923 & 484,295 \\
\hline 2010 & 213,056 & 132,336 & 131,782 & 477,174 \\
\hline 2011 & 208,885 & 119,465 & 141,685 & 470,035 \\
\hline 2012 & 216,670 & 114,001 & 152,168 & 482,839 \\
\hline 2013 & 224,006 & 119,572 & 157,751 & 501,329 \\
\hline
\end{tabular}

Fuente: Elaborado de cifras del 2013 Yearbook of Immigration Statistics. U.S. Department of Homeland Security.

*Admisiones de no inmigrante se refieren a las llegadas de las personas que están autorizadas a permanecer en los Estados Unidos por un períado limitado de tiempo. La mayoría de los no inmigrantes entran a los Estados Unidos como turistas o viajeros de negocios, pero algunos vienen a trabajar, estudiar o participar en programas de intercambio cultural

Estados Unidos de América realiza acciones de aplicación a poblaciones que violan la ley de inmigración y nacionalidad, entre ellas están la inadmisibilidad, las aprehensiones, expulsiones y devoluciones. Los aprehendidos han cometido faltas y que pueden llegar a ser sancionados. Los nativos de Guatemala y Honduras tienen el mayor número de eventos de aprehensión (Cuadro $\mathrm{N}^{\circ} 11$ ).

Cuadro ${ }^{0} 11$. EUA: aprehendidos ciudadanos de países del Triángulo Norte de Centroamérica, años fiscales 2008-2013

\begin{tabular}{|c|c|c|c|c|}
\multirow{2}{*}{ Años } & \multicolumn{3}{|c|}{ Países } & \multirow{2}{*}{ Total } \\
\cline { 2 - 4 } & Guatemala & El Salvador & Honduras & \\
\hline 2008 & 33,690 & 27,153 & 33,777 & 94,620 \\
\hline 2009 & 34,992 & 27,741 & 32,901 & 95,634 \\
\hline 2010 & 39,050 & 29,911 & 32,501 & 101,462 \\
\hline 2011 & 41,708 & 27,652 & 31,189 & 100,549 \\
\hline 2012 & 57,486 & 38,976 & 50,771 & 147,233 \\
\hline 2013 & 73,208 & 51,226 & 64,771 & 188,591 \\
\hline
\end{tabular}

Fuente: Elaborado de cifras del 2013 Yearbook of Immigration Statistics. U.S. Department of Homeland Security 
Las expulsiones surgen de movimientos de extranjero inadmisible o en condición de deportable fuera de los Estados Unidos, a su vez cuando se expulsa a un extranjero tiene consecuencias administrativas o penales impuestas a la posterior reentrada debido al hecho de la eliminación. Guatemala y Honduras tienen el mayor número de ciudadanos expulsados de los tres países que en los últimos años es creciente (Cuadro $\mathrm{N}^{\circ} 12$ ).

CUADRO N ${ }^{0} 12$. EUA: expulsados ciudadanos de países del Triángulo Norte de Centroamérica, años fiscales 2008-2013

\begin{tabular}{|c|c|c|c|c|}
\multirow{2}{*}{ Años } & \multicolumn{3}{|c|}{ Países } & \multirow{2}{*}{ Total } \\
\cline { 2 - 4 } & Guatemala & El Salvador & Honduras & \\
\hline 2008 & 33,690 & 27,153 & 33,777 & 94,620 \\
\hline 2009 & 34,992 & 27,741 & 32,901 & 95,634 \\
\hline 2010 & 39,050 & 29,911 & 32,501 & 101,462 \\
\hline 2011 & 41,708 & 27,652 & 31,189 & 100,549 \\
\hline 2012 & 57,486 & 38,976 & 50,771 & 147,233 \\
\hline 2013 & 73,208 & 51,226 & 64,771 & 188,591 \\
\hline
\end{tabular}

Fuente: Elaborado de cifras de 2013 Yearbook of Immigration Statistics. U.S. Department of Homeland Security

\subsubsection{Estadísticas de la Oficina de Aduanas $y$} Protección Fronteriza de los EUA (CBP)

Esta agencia federal es responsable de impedir la entrada de terrorista y armas, capturar personas que traten de entrar ilegalmente a ese país, detener el flujo de drogas ilegales, contrabando y otras actividades de protección de intereses norteamericanos. De las 486,651 detenciones en todo el país, 468,407 de esas eran de individuos de México, El Salvador, Guatemala y Honduras, y casi todas las aprehensiones fueron a lo largo de la frontera suroeste. En el año fiscal 2014, la CBP ha aprehendido 66,638 los nacionales de El Salvador, 81,116 de Guatemala, 91,475 nacionales de Honduras y 229,178 ciudadanos de México. Para el 2015 la CBP aprehendió a 43,654 nacionales de El Salvador, 57,160 de Guatemala y 33,848 de Honduras, de México se aprendieron a 188,122 personas (United States Department Homeland, 2016).

En los últimos años, específicamente a partir del 2012 la CBP detectó un aumento en la aprehensión de extranjeros menores no acompañados (de 0 a 17 años) especialmente los procedentes de los países del triángulo norte de Centroamérica en la frontera sudoeste, específicamente en el Valle del Río Grande, que en período corto de tiempo, se elevaron de niveles históricamente bajos a presentar un importante flujo. En el 2014 se reporta un total el mayor nivel de 51,705 niños no acompañados de los tres países de la subregión y 15,634 de México, en los siguientes años hay una tendencia al descenso (Cuadro $\mathrm{N}^{\circ} 13$ ).
Cuadro $\mathrm{N}^{0}$ 13. EUA: menores de edad* no acompañados encontrados de los principales países por años fiscales, 2009-2016

\begin{tabular}{|c|c|c|c|c|}
\hline \multirow{2}{*}{ Años } & \multicolumn{5}{|c|}{ Países } \\
\cline { 2 - 5 } & El Salvador & Guatemala & Honduras & México \\
\hline 2009 & 1,221 & 1,221 & 968 & 16,114 \\
\hline 2010 & 1,910 & 1,517 & 1,017 & 13,724 \\
\hline 2011 & 1,394 & 1,565 & 974 & 11,768 \\
\hline 2012 & 3,314 & 3,835 & 2,997 & 13,974 \\
\hline 2013 & 5,990 & 8,068 & 6,747 & 17,240 \\
\hline 2014 & 16,404 & 17,057 & 18,244 & 15,634 \\
\hline 2015 & 9,389 & 13,589 & 5,409 & 11,012 \\
\hline 2016 & 6,621 & 8,329 & 3,608 & 4,658 \\
\hline
\end{tabular}

Fuente: Oficina de Aduanas y Protección Fronteriza de EUA. Años fiscales 2009-2015. Oct 1-Feb. 29, 2016. *Menores de 18 años

La llamada "crisis humanitaria" de los niños migrantes no acompañados también incluyó la aprehensión de unidades familiares de menores que fueron detenidos con otro familiar, estos flujos fueron más significativos de los nativos de los países del triángulo norte centroamericano (Cuadro $\mathrm{N}^{\circ}$ 14).

Cuadro $N^{0} 14$. EUA: unidades familiares * aprehendidas encontradas de los principales países por año fiscal, 2015-2016

\begin{tabular}{|c|c|c|}
\hline País & 2015 & 2016 \\
\hline El Salvador & 10,872 & 9,314 \\
\hline Guatemala & 12,820 & 8,823 \\
\hline Honduras & 10,671 & 6,965 \\
\hline México & 4,276 & 1,312 \\
\hline
\end{tabular}

El Instituto de Políticas de Migración (MPI) ha elaborado un completo estudio estadístico y de perfiles de los migrantes del Triángulo Norte de Centroamérica deportados de México y Estados Unidos de América, donde compatibiliza y ajusta los datos de ambas fuentes para ofrecer las cifras más cercanas a la realidad migratoria (Cuadro $\mathrm{N}^{\circ} 15$ ). Admite que casi un millón de personas de la subregión del triángulo norte han sido aprehendidas por las autoridades de EUA y México en los últimos años y que 800 mil han sido deportadas, muchos de ellos niños, entre el 2010-2014 han sido aprehendidos 130 mil menores y 40 mil deportados (Rodrigo \& Rietig, 2015). 
Cuadro $\mathrm{N}^{\circ} 15$. Deportados de EUA y México nacidos en países del Triángulo Norte de Centroamérica según año fiscal, 2010-2015, en miles

\begin{tabular}{|c|c|c|}
\multirow{2}{*}{ Años } & \multicolumn{2}{|c|}{ Países de deportación } \\
\cline { 2 - 3 } & EUA & México \\
\hline 2010 & 80 & 63 \\
\hline 2011 & 75 & 58 \\
\hline 2012 & 94 & 74 \\
\hline 2013 & 107 & 77 \\
\hline 2014 & 122 & 87 \\
\hline $2015^{*}$ & & 156 \\
\hline
\end{tabular}

Fuente: Tomado de Dominguez Villegas, Rodrigo and Victoria Rietig. 2015. Migrants Deported from the United States and Mexico to the NorthermTriangle: A Statistical and Socioeconomic Profile. Washington, DC: Migration Policy Institute. Notes: Data on deportations from the United States in 2014 include only removals. Data for FY 2015 are not yet available to estimate projections. FY 2015 data for Mexico represent an MPI projection based on the proportion of annual deportations to countries in the Northern Triangle observed during the first six months of the fiscal year. Historically, these months have accounted or 44 percent of annual deportations, and INM reported 6B,777 deportations in the first six months of FY 2015. Sources: U.S. Data for the United States for FY 2014 from Immigrations and Customs Enforcement (ICE), "FY 2014 ICE Immigration Removals," accessed April 23, 2015,

wwwice gov/removal-statistics; for FY 2010-13 from Department of Homeland Security (DHS) Office of Immigration Statistics (OIS), Yearbook of Immigration Statistics:

2013 Washington, DC: DHS, 2013), www.dhs-govyearbook-immigration-statistics-2013-enforcement-actions MPI calculations for FY 2015 and FY 2010-14 for México from INM, "Boletín Mensual de Estadísticas Migratorias," FY 2009-15

\subsubsection{Estimaciones de migración no autorizada}

Los tanques de pensamiento e instituciones estatales han elaborado estimaciones del número de poblaciones no autorizadas que residen en los Estados Unidos de América, todas ellas calculan entre 1.4 a 1.6 millones de personas nativas de los países de la subregión que no están autorizados para vivir en el país del norte. Guatemala y El Salvador tienen la mayor cantidad de población que está en situación irregular (Cuadro $\mathrm{N}^{\circ} 16$ ). Estas estimaciones para el año 2012-2013 representarían alrededor de un 35$40 \%$ de la población de origen y $52-59 \%$ de la nacida en los países del triángulo norte que reside en Estados Unidos en esos años (Rosenblum \& Ruiz Soto, 2015).

\subsubsection{Estimaciones de aplicantes a programa DACA}

La administración del Presidente Barack Obama ha desarrollado programas para diferir las acciones de aplicación de inmigración contra las personas indocumentadas que viven en los Estados Unidos, el primero dirigido a la Acción Diferida para los padres de los estadounidenses y residentes legales permanentes (DAPA), y el segundo llamado Acción Diferida para los Llegados en la Infancia (DACA), que comenzó en 2012 y se amplió en 2014 (DACA-Plus). El Instituto de Políticas de Migración (MPI) ha hecho estimaciones de poblaciones elegibles al programa DACA que podrían alcanzar a 161 mil personas en el orden por su volumen de Guatemala, El Salvador y Honduras, pero las tasas de aplicación basadas en los datos administrativos de aplicación giran alrededor del 42-68\% para los tres países en su conjunto, aunque hay diferencias que favorecerían a los nacidos en El Salvador, Honduras y en menor medida a los de Guatemala (Cuadro $\mathrm{N}^{\circ} 17$ ).

Cuadro $\mathrm{N}^{0} 16$. EUA: comparación de estimaciones recientes de población no autorizada por fuente de datos según país de nacimiento del Triángulo Norte de Centroamérica

\begin{tabular}{|l|c|c|c|c|}
\hline & \multicolumn{3}{|c|}{ Países } & \multirow{2}{*}{ Total } \\
\cline { 2 - 4 } & Guatemala & El Salvador & Honduras & \\
\hline $\begin{array}{l}\text { Instituto de } \\
\text { Políticas de } \\
\text { Migración } \\
\text { MPI 2013 }\end{array}$ & 704,000 & 436,000 & 317,000 & $1,457,000$ \\
\hline $\begin{array}{l}\text { Centro de } \\
\text { Estudios } \\
\text { Migratorios } \\
\text { 2013 }\end{array}$ & 518,000 & 588,000 & 324,000 & $1,430,000$ \\
\hline $\begin{array}{l}\text { PEW Centro } \\
\text { de Investiga- } \\
\text { ción Hispana } \\
\text { 2012 }\end{array}$ & 525,000 & 675,000 & 350,000 & $1,550,000$ \\
\hline $\begin{array}{l}\text { Departamento } \\
\text { de Seguridad } \\
\text { Nacional } \\
\text { 2012 }\end{array}$ & 525,000 & 675,000 & 350,000 & $1,550,000$ \\
\hline
\end{tabular}

Fuente: Tomado de Rosenblum, Mark R. \& Ruiz Soto, Ariel G. 2015. An Analysis of Unauthorized Immigrants in the United States by Country and Region Birth, Washington D.C., Migration Policy Institute. * Las estimaciones del MPI 2013 excluye a los amparados por TPS de El Salvador y Honduras, mientras en las otras estimaciones de las otras fuentes los incluyen

Cuadro $\mathrm{N}^{0}$ 17. EUA: estimaciones de tasas de aplicación de DACA entre jóvenes inmediatamente y potencialmente elegible de los páses del Triángulo Norte de Centroamérica, 2009-13

\begin{tabular}{|c|c|c|c|c|c|c|}
\hline \multirow[b]{2}{*}{ País } & \multicolumn{3}{|c|}{ MPI Estimaciones de DACA: poblaciones elegibles } & \multirow[b]{2}{*}{$\begin{array}{c}\text { Aplicaciones } \\
\text { DACA(USCIS } \\
\text { datos } \\
\text { administrativos } \\
\text { de } 31 \text { marzo de } \\
2015 \text { ) }\end{array}$} & \multicolumn{2}{|c|}{ Estimaciones de tasas de aplicación (\%) } \\
\hline & $\begin{array}{c}\text { Elegible } \\
\text { inmediatamente }\end{array}$ & $\begin{array}{l}\text { Elegible } \\
\text { pero por } \\
\text { Educación }\end{array}$ & $\begin{array}{l}\text { Potencialmente } \\
\text { elegible: } \\
\text { Inmediatamente } \\
\text { + pero por } \\
\text { educación }\end{array}$ & & $\begin{array}{c}\text { Potencialmente } \\
\text { Elegible }\end{array}$ & $\begin{array}{l}\text { Inmediatamente } \\
\text { Elegible }\end{array}$ \\
\hline Guatemala & 45,000 & 37,000 & 83,000 & 20,000 & $24 \%$ & $44 \%$ \\
\hline El Salvador* & 31,000 & 12,000 & 44,000 & 28,000 & $65 \%$ & $91 \%$ \\
\hline Honduras* & 23,000 & 10,000 & 34,000 & 19,000 & $56 \%$ & $81 \%$ \\
\hline Total & 99,000 & 59,000 & 161,000 & 67,000 & $42 \%$ & $68 \%$ \\
\hline
\end{tabular}

Fuente: MPI Analys of 2009-13 ACS and 2008 SIPP data by Hammar, Bachmeier, and Van Hook; USCIS. Number of I-82ID. Consideration of Deferred Action for Childhood Arrivals by Fiscal Year, Quarter, Intake, Biometrics and case Status: 2012-2015 (March 31).

https://www.uscis.gov/sites/default/files/USCIS/Resources/Reports\%20and\%20.Studies/Immigration\%20Forms\%20Data/Naturalization\%20Data/I821d_performancedata_fy2 2015_qut2.pdf Nota: La población elegible inmediatamente incluye a los inmigrantes no autorizados de 15 años y más de edad, año de llegada, la presencia continua y los requisitos de educación para el programa DACA 2012. La población potencialmente elegible inchuye a los inmigrantes no autorizados que cumplan la misma edad, año de llegada y las requisitos de presencia continua. pero que no tienen un grado de escuela secundaria o equivalente, $y$ no aslaba inscrito en la escuela. Estas estrimaciones no inc

*Sumatoria y estimación de tasa de aplicación para países del triángulo norte de Centroamérica no incluida en cuadro original 


\section{Conclusiones}

- Los censos de población y las encuestas especiales de los países de origen producen datos para estimaciones directas que subvalúan los niveles de migración al exterior, al menos generan información de los territorios de la emigración y de algunas características de los migrantes. Es necesaria hacer estimaciones indirectas de los censos y encuestas.

Las estadísticas migratorias de carácter administrativo en los países de origen son una veta de posibilidades de explorar los diferenciales de entradas y salidas, principalmente cuando se realizan por las estaciones migratorias, con las desventajas de no medición terrestre por cruces de fronteras irregulares. Las instituciones gubernamentales encargadas del control migratorio no divulgan suficiente información que podría ayudar a mejores estimaciones de la migración.

- En los países en tránsito, específicamente México hay grandes esfuerzos en la cuantificación de las poblaciones que cruzan por ese país en su ruta hacia el norte. Las EMIF Sur permiten conocer los flujos y características de los emigrantes del triángulo norte de Centroamérica. Las estimaciones de los académicos mejicanos de la migración en tránsito de la subregión por México no solo han permitido cuantificar el flujo, sino también identificar la magnitud a través del tiempo que responde a diversas causas en los países de origen. Las estadísticas migratorias mejicanas presentan un panorama de comprobación de que ese país es utilizado principalmente como tránsito y no como destino de la emigración de la subregión, al observar los niveles de presentación ante autoridades migratorias, devolución y retornos asistidos de poblaciones, incluso de menores de edad y que cada año se profundiza más con los resultados de los planes en la frontera sur de México diseñados especialmente para la detención de migrantes que van hacia el norte.

En el país de destino existe una amplia gama de fuentes de información que van desde los censos de población, encuestas, registros administrativos migratorios y estimaciones de poblaciones no autorizadas y de aplicación de programas espaciales para tener oportunidades de regularización. Cada una de ellas tiene sus potencialidades y objetivos que pueden ser utilizados para nuestros intereses, en los primeros básicamente de los niveles y características sociodemográficas de las poblaciones residentes en ese país. En los registros migratorios de los flujos, viajeros, aprehensiones y devoluciones. En las estimaciones básicamente realizadas por tanques de pensamiento que plantean la idea de magnitud de residentes no autorizados y de quienes podrían obtener un beneficio migratorio.

De las fuentes de datos e información se desprenden estimaciones en los países de origen que necesitan depurarse, reforzarse y completarse, en los países en tránsito con estimaciones de cien a doscientos mil personas que anualmente recorren el territorio mejicano y en el país de destino que incluye a una población residente de origen (4.2 millones) y una residente nacida de los países de la subregión (2.8 millones), las llegadas en el último año entre 60-80 mil personas (ACS 2014). A su vez hay una gran cantidad de personas aprehendidas (1 millón) y deportadas (800 mil) de EUA y México hacia sus países de origen en los últimos cinco años.

Finalmente, es necesario apuntar que la migración del triángulo norte de Centroamérica aunque en sus orígenes estuvo influenciada por los conflictos armados en la región, caminó a través del tiempo a causas que se originan en los países de origen en la presión de poblaciones marginadas y excluidas de oportunidades y se agregan los desequilibrios generados de las políticas gubernamentales, especialmente las económicas, los desastres naturales que han sumado causas, pero en los últimos años el recrudecimiento de la violencia ha generado nuevas oleadas que aunque no es la causa principal en la búsqueda del sueño americano contribuye y todo eso se comprueba con las cifras de diversas fuentes, pero se agudizan las medidas de detención de migrantes en la fronteras estadounidense, pero más en la frontera del país en tránsito. 


\section{Bibliografía}

- Colegio de la Frontera Norte, Secretaría de Gobernación, Secretaría de Trabajo y Previsión Social, Secretaría de Relaciones Exteriores. (2014). Encuesta sobre Migración en la Frontera Sur de México, EMIF Sur, Informe Anual de Resultados 2013. México D.F.

- DIGESTYC. (2015). Encuesta de Hogares de Propósitos Multiples, EHPM 2014 El Salvador. San Salvador: DIGESTYC.

- DIGeSTYC, D. G. (2009). VI Censo de Población y $V$ de Vivienda 2007, Tomo IV: Volumen I - Municipios, Características Generales. San Salvador.

- Flores Fonseca, M. A. (2015). Cambios y Desafíos de Centroamérica. XXX Congreso Latinaomericano de Sociología. San José: ALAS.

- INE. (2003). Características de la Población y de los Locales de Habitación Censados . Guatemala: INE.

- ITAM. (2014). Migración Centroamericana en Tránsito por México hacia Estados Unidos: Diagnóstico y Recomendaciones. Hacia una visión integral, regional y de responsabilidad compartida. México D.F.: Instituto Tecnológico Autónomo de México.

- OIM. (2015). Informe sobre las Migraciones en el Mundo 2015. Los migrantes y las ciudades: Nuevas colaboraciones para gestionar la movilidad. Ginebra: Organización Internacional de las Migraciones.

- Rodrigo, D., \& Rietig, V. (2015). Migrant deported from the United States and Mexico to the Northern Triangle: A statistical and Socioeconmic Profile. Washington D.C.: Migration Policy Institute.

- Rosenblum, M., \& Ruiz Soto, A. (2015). An Analysis of Unauthorized Immigrants in the United States by Country and Region Birth. Washington D.C.: Migration Policy Institute.

- Ruggles, S., Genadek, K., Goeken, R., Grover, J., \& Sobek, M. (2015). Integrated public Use Microdata series: Version 6.0. Minneapolis, Minnesota, USA.

- United States Department Homeland. (2016). CBP Border Security Report Fiscal Year 2015. Washington, D.C.: CBP.

- United States Department of Homeland. (2015). 2013 Yaerbook of Inmigration Statistics. Washington D.C: Department of Homeland Security, Office of Immigration Statistics 